ACCEPTED MANUSCRIPT

\title{
Rigidity of MHD equilibria to smooth incompressible ideal motion near resonant surfaces
}

To cite this article before publication: David Pfefferlé et al 2020 Plasma Phys. Control. Fusion in press https://doi.org/10.1088/1361-6587/ab8ca3

\section{Manuscript version: Accepted Manuscript}

Accepted Manuscript is "the version of the article accepted for publication including all changes made as a result of the peer review process, and which may also include the addition to the article by IOP Publishing of a header, an article ID, a cover sheet and/or an 'Accepted

Manuscript' watermark, but excluding any other editing, typesetting or other changes made by IOP Publishing and/or its licensors"

This Accepted Manuscript is @ 2020 IOP Publishing Ltd.

During the embargo period (the 12 month period from the publication of the Version of Record of this article), the Accepted Manuscript is fully protected by copyright and cannot be reused or reposted elsewhere.

As the Version of Record of this article is going to be / has been published on a subscription basis, this Accepted Manuscript is available for reuse under a CC BY-NC-ND 3.0 licence after the 12 month embargo period.

After the embargo period, everyone is permitted to use copy and redistribute this article for non-commercial purposes only, provided that they adhere to all the terms of the licence https://creativecommons.org/licences/by-nc-nd/3.0

Although reasonable endeavours have been taken to obtain all necessary permissions from third parties to include their copyrighted content within this article, their full citation and copyright line may not be present in this Accepted Manuscript version. Before using any content from this article, please refer to the Version of Record on IOPscience once published for full citation and copyright details, as permissions will likely be required. All third party content is fully copyright protected, unless specifically stated otherwise in the figure caption in the Version of Record.

View the article online for updates and enhancements. 


\title{
Rigidity of MHD equilibria to smooth incompressible ideal motion near resonant surfaces
}

\author{
David Pfefferlé, ${ }^{1}$ Lyle Noakes, ${ }^{1}$ and Yao Zhou ${ }^{2}$ \\ ${ }^{1}$ The University of Western Australia, 35 Stirling Highway, Crawley WA 6009, Australia \\ ${ }^{2}$ Princeton Plasma Physics Laboratory, Princeton, NJ 08543, USA
} (Dated: April 21, 2020)

\begin{abstract}
In ideal MHD, the magnetic flux is advected by the plasma motion, freezing flux-surfaces into the flow. An MHD equilibrium is reached when the flow relaxes and force balance is achieved. We ask what classes of MHD equilibria can be accessed from a given initial state via smooth incompressible ideal motion. It is found that certain boundary displacements are formally not supported. This follows from yet another investigation of the Hahm-Kulsrud-Taylor (HKT) problem, which highlights the resonant behaviour near a rational layer formed by a set of degenerate critical pointsin the flux-function. When trying to retain the mirror symmetry of the flux-function with respect to the resonant layer, the vector field that generates the volume-preserving diffeomorphism vanishes at the identity to all order in the time-like path parameter.

Keywords: MHD equilibrium; frozen-in flux; volume-preserving diffeomorphism; generating functions
\end{abstract}

\section{INTRODUCTION}

Renewed interest in stellarator design has sparked questions on the existence and accessibility of threedimensional magneto-hydrodynamic (MHD) equilibria with "good" nested flux-surfaces $[1-5]$. Several numerical tools exist to obtain three-dimensional MHD equilibria by means of variational principles [6-8], initial value problems $[9,10]$, iterative methods $[11,12]$, metriplectic formulations [13], analytic expansions around a given magnetic axis $[14,15]$. These methods aspire to produce and optimise the magnetic fields so that the field-lines lie on toroidally nested flux-surfaces [16, 17], which is the basis of plasma confinement in magnetic fusion devices such as tokamaks and stellarators [18].

Restricted to flux-surfaces, the magnetic field is an integrable Hamiltonian vector field [19-21]. Under the assumption of translational and/or rotational symmetry (isometries), the MHD equilibrium problem reduces to a two-dimensional elliptic PDE for the scalar fluxfunction, called the Grad-Shafranov equation [22, 23]. Flux-surfaces naturally correspond to the level sets of the flux-function extruded to surfaces along the direction of symmetry.

Without explicit symmetry, it can be proven, whenever the plasma current is not everywhere parallel to the magnetic field, that field-lines lie on nested tori [24, Theorem 7]. However, the embedding of these topological tori may be extremely complicated (knotted) in real space. The theorem also does not inform on the existence nor on the accessibility of such equilibrium configurations. On the other hand, force-free fields correspond to minimum energy solutions of the boundary value problem. The behaviour of their field-lines is known to be chaotic even in the simplest three-dimensional instances $[25,26]$. Three-dimensional MHD equilibria (within the class of smooth magnetic fields) whose field-lines lie on simple nested flux-surfaces are thus rather exceptional, fine-tuned solutions.
The goal of this paper is to illustrate an essential difficulty in smoothly deforming an initial configuration with nested flux-surfaces through a family of MHD equilibria to reach a target three-dimensional configuration with equivalent (diffeomorphic) flux-surfaces. In the language of toroidal fusion plasmas, the issue is that flux-surfaces with periodic field-lines (rational rotational transform) are sensitive to resonant perturbations. Even the smallest amplitude leads to the formation of magnetic islands in the vicinity of resonant surfaces. Larger resonant perturbations often result in the chaotic meandering of magnetic field-lines, in which case flux-surfaces cease to exist. The transition from periodic to chaotic dynamics of magnetic field-lines is well understood in the context of classical Hamiltonian perturbation theory [21, 27, 28]. Whether resonance can be avoided in order to form a smooth sequence of MHD equilibrium states is a different line of inquiry, closely related to the study of stationary solutions to Euler equations and their properties [29, 30].

In mathematical terms, the presence of degenerate critical points in the flux-function prevents us to claim that the topology of level sets remains fixed under arbitrary boundary deformations. Rigidity is shown by proving nonexistence of smooth solutions within the assumptions of the so-called Hahm-Kulsrud-Taylor (HKT) problem [31]. The core obstruction to deforming solutions in the simpler HKT example is merely exacerbated in the three-dimensional MHD equilibrium problem due to geometry and non-linearities. It is important to recognise that known methods of solutions to the HKT problem $[2,5,31-36]$ depart from a strictly ideal treatment by either introducing finite resistivity or discontinuities in the gradient of the flux-function. In the latter case, the smoothness of the plasma displacement and the accessibility from a smooth initial configuration are compromised. Our work invites for a more conscientious investigation of non ideal-MHD effects in the modelling of three-dimensional MHD equilibrium.

The paper is organised as follows. In section II, the

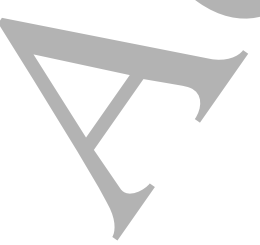


MHD equilibrium problem is set up in the simpler case of a slab with an ignorable coordinate. In section III, elements of differential geometry are introduced to show that the force-balance condition can be cast as the vanishing of a Poisson-bracket between the flux-function and its Laplacian. In section IV, the frozen-in condition of ideal MHD is identified with the precomposition of the flux-function by a diffeomorphism (pullback). The requirement for smooth incompressible ideal motion naturally follows from the preservation of the Poissonbracket (symplectomorphism). The idea is then to generate a family of diffeormorphisms via a sequence of $t$ dependent divergence-free Eulerian velocity fields in order to smoothly map an initial equilibrium state into another (by constructing an isotopy). Examples of oneparameter subgroups of diffeomorphisms are shown to illustrate the challenge of retaining force-balance beyond linear order in the $t$-parameter. In section $\mathrm{V}$, we propose a method of solution based on a hierarchy of linear inhomogeneous PDE systems to recover the flux-function and the potential function as a Taylor series in the $t$ parameter. There are no real obstruction to applying this procedure to the case of a vacuum field as initial configuration. However, we show that there are no smooth Eulerian velocity fields that can deform the level sets of the initial flux-function of the HKT problem and conform with a mirror-symmetric boundary condition. With this result, we conclude in section VI that MHD equilibria with degenerate critical points in the flux-function, namely a rational surfaces in the three-dimensional case, are rigid to large classes of smooth incompressible ideal deformations.

\section{THE GRAD-SHAFRANOV EQUATION IN A SLAB}

We avoid complexities due to geometry by working in the much simpler setting of a slab, $(x, y, z)$, where $x$ plays the role of a radial variable, $y$ the periodic poloidal angle and $z$ the ignorable toroidal angle. As illustrated in Figure 1, the slab system is the local limit of a helical flux tube in the vicinity of a resonant flux-surface [1$3,5,31,33,34,36-39]$. A major obstruction to accessing equivalent equilibrium states through ideal motion already exists in this case and is carried over to toroidal and other geometries by elliptic regularity of the differential operators.

By symmetry along the $z$-axis, the form of the vector potential is, up to a gauge term, $\boldsymbol{A}=A_{y}(x, y) \nabla y+$ $\Psi(x, y) \nabla z$ and the magnetic field $\boldsymbol{B}=I(x, y) \nabla z+\nabla \Psi \times$ $\nabla z$. Throughout the paper, $\Psi(x, y)$ will be called the flux-function. A stationary solution (without flow) to the ideal MHD equations is a configuration such that $\boldsymbol{J} \times \boldsymbol{B}=\nabla p$, where $p(x, y)$ is identified as the plasma pressure and $\boldsymbol{J}=\nabla \times \boldsymbol{B}$ the current density. In this representation, a state of force-balance is achieved when the gradient of the pressure $p(x, y)$ and the gra-

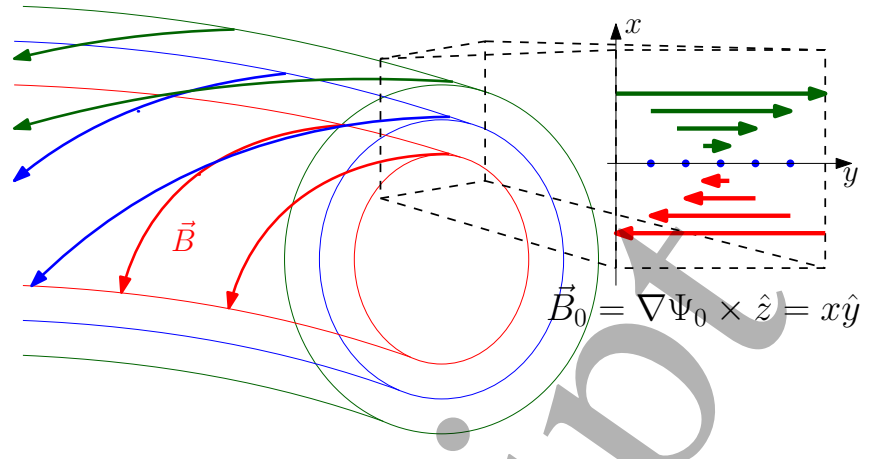

Figure 1. Local limit of a helical flux-tube in the/vicinity of a resonant surface (whose rotational transform is rational). The $x$-coordinate of the slab system is normal to the flux surfaces (radial) with origin at the rational layer. The $z$ coordinate points in the (ignorable) helical direction and the $y$-coordinate in the poloidal direction. Close to the resonant surface, the helical magnetic field expressed in slab coordinates $B_{0}=\nabla \times\left(\Psi_{0} \nabla z\right)$ is approximately linear in $x$. This qualitative behaviour justifies the study of the quadratic fluxfunction $\Psi_{0}=\frac{1}{2}\left(1-x^{2}\right)$ as an initial equilibrium configuration of the HKT problem.

dient of the longitudinal current (a.k.a the guide field) $I(x, y)$ are collinear with the gradient of the flux-function, $\nabla p \times \nabla \Psi=0$ and $\nabla I \times \nabla \Psi=0$, i.e. $\nabla p=p^{\prime}(x, y) \nabla \Psi$ and $\nabla I=I^{\prime}(x, y) \nabla \Psi$. By virtue of the ignorable $z$ coordinate, the MHD equilibrium problem reduces to the 2D nonlinear elliptic Grad-Shafranov equation

$$
\Delta \Psi=\bar{I} \bar{I}^{\prime}+\bar{p}^{\prime}=V^{\prime}(\Psi),
$$

where $\bar{I}: \mathbb{R} \rightarrow \mathbb{R}$ and $\bar{p}: \mathbb{R} \rightarrow \mathbb{R}$ are functions of the flux such that $I(x, y)=\bar{I}(\Psi(x, y))$ and $p(x, y)=\bar{p}(\Psi(x, y))$, and $\Delta=\nabla \cdot \nabla$ is the Laplacian operator. The formulation of the MHD equilibrium problem in a slab is similar to that of reduced MHD [40, 41]. The important difference is that the guide-field in reduced MHD is constant, $I(x, y)=I_{0}$.

A convenient way to rephrase the force-balance condition and eliminate the arbitrary pressure and current functions is

$$
\nabla \times(\Delta \Psi \nabla \Psi)=0 .
$$

Remark. The Grad-Shafranov equation (1) implies equation (2). The converse is in general not true, e.g. $\Delta \Psi$ piecewise-constant.

We will consider throughout the paper that an MHD equilibrium is determined by a flux-function $\Psi$ that satisfies equation (2). For example, harmonic functions are special cases describing vacuum fields, $\Delta \Psi=0$. Solutions to the uniform current density or constant pressuregradient Poisson equation, $\Delta \Psi=$ const, form another particular class, comprising the initial configuration of the HKT problem, $\Psi_{0}(x, y)=\frac{1}{2}\left(1-x^{2}\right)$. Taylor-relaxed fields [42] are a third kind of solutions satisfying the Helmholtz equation $\Delta \Psi=-\mu^{2} \Psi$, and coincide with the eigenfunctions of the Laplacian. 


\section{DIFFERENTIAL GEOMETRIC SETTING}

Useful information and structure is obtained by rephrasing the problem in the language of differential geometry. The advantage is that part of the conclusions will be independent of the specific choice of coordinates and straightforwardly transposable to more complicated geometries such as the toroidal case, as well as threedimensional equilibria.

Briefly, let $M=\mathbb{R} \times S^{1}=\left\{\left(x, e^{i y}\right) \mid x \in \mathbb{R}, y \in\right.$ $\mathbb{R}\}$. We equip $M$ with the standard volume form $\omega=d x \wedge d y$ and the standard Euclidean metric $\langle X, Y\rangle(p)=X^{x}(p) Y^{x}(p)+X^{y}(p) Y^{y}(p)$, where $X f(p)=$ $X^{x}(p) \partial_{x} f(p)+X^{y}(p) \partial_{y} f(p)$ represents the vector field $X$ acting as a differential operator on the smooth function $f: M \rightarrow \mathbb{R}$. Appendix A summarises the definitions and conventions used throughout the document.

Remark. We will identify $d y$ as the closed but misleadingly not exact one-form on $S^{1}$ such that $\oint d y=2 \pi m$ where the integer $m \in \mathbb{Z}$ depends only the closed integration path.

The volume form $\omega$ plays the role of a symplectic form on this two-dimensional manifold, enabling the identification of Hamiltonian vector fields (see Appendix B): given a smooth function $F: M \rightarrow \mathbb{R}$, there is a unique vector field $X_{F}$ such that

$$
i_{X_{F}} \omega=d F, \quad \text { or } \quad X_{F}^{b}=-\star d F .
$$

Hamiltonian vector fields are divergence-free in our setup, because $\left(\operatorname{div} X_{F}\right) \omega:=£_{X_{F}} \omega=d i_{X_{F}} \omega=d^{2} F=0$. This property means that the flow of $X_{F}$ is a one-parameter family of volume-preserving diffeomorphisms on $M$ (simultaneously symplectomorphisms), under which the volume form is preserved (advected) along $X_{F}$. Furthermore, since $X F=£_{X_{F}} F=i_{X_{F}} d F=i_{X_{F}} i_{X_{F}} \omega=0$, the Hamiltonian $F$ is preserved along $X_{F}$ or, equivalently, the Hamiltonian vector field $X_{F}$ is tangential to curves of constant $F$.

As differential operators, Hamiltonian vector fields can be used to define an anti-symmetric bilinear operation on smooth functions $F$ and $G$ by

$$
\{F, G\}:=-£_{X_{F}} G=\omega\left(X_{F}, X_{G}\right)
$$

which is the same as $\{F, G\} \omega=d F \wedge d G$ in our twodimensional setting where $\omega$ is also the volume form. This product rule qualifies as a Poisson-bracket (see Result B.1). In local coordinates, this Poisson-bracket is simply computed as

$$
\{F, G\}=\partial_{x} F \partial_{y} G-\partial_{y} F \partial_{x} G=(\nabla F \times \nabla G) \cdot \nabla z .
$$

In this context, the flux-function $\Psi: M \rightarrow \mathbb{R}$ is the Hamiltonian for the poloidal (or helical) magnetic field $B$, obtained via $i_{B} \omega=d \Psi$ or $B^{b}=-\star d \Psi$. This construction automatically ensures that the magnetic field is tangential to curves of constant $\Psi$, called flux-levels. We will refer to the quantity $d \Psi$ as the magnetic one-form (magnetic flux density).
Remark. In local coordinates $B=\partial_{y} \Psi \partial_{x}-\partial_{x} \Psi \partial_{y}$ or equivalently $\boldsymbol{B}=\nabla \times(\Psi \nabla z)=\nabla \Psi \times \nabla z$ is the poloidal (or helical) component of the magnetic field in $3 \mathrm{D}$, as in Figure 1.

The force-balance condition of equation (2) is identified as the Poisson-commutation of the flux-function with its Laplacian

$$
d(\Delta \Psi d \Psi)=d(\Delta \Psi) \wedge d \Psi=0 \Longleftrightarrow\{\Delta \Psi, \Psi\}=0
$$

where $\Delta=\delta d+d \delta=(\delta+d)^{2}$ is the so-called Laplacede Rham operator and $\delta=-\star d \star$ is the codifferential. Another way of viewing equation (5) is $B \Delta \Psi=\mathscr{E}_{B}^{\prime} \Delta \Psi=$ 0 , namely that the magnetic field is tangential to the level sets of the Laplacian of the flux-function.

\section{FROZEN-IN CONDITION AND SMOOTH INCOMPRESSIBLE IDEAL MOTION}

An MHD equilibrium $\Psi$ is said to be accessible through ideal motion from an initial MHD equilibrium $\Psi_{0}$ if there exists a diffeomorphism (smooth map with smooth inverse) $\varphi: M \rightarrow M$ such that the following diagram commutes

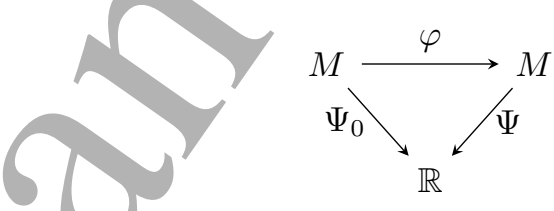

The flux-functions are effectively related by precomposition with the inverse diffeomorphism (pullback-byinyerse, see Appendix A)

$$
\Psi=\Psi_{0} \circ \varphi^{-1}=\varphi^{-1^{*}} \Psi_{0}=: \varphi_{*} \Psi_{0} .
$$

The magnetic one-forms are related in the same way, $d \Psi=d \varphi_{*} \Psi_{0}=\varphi_{*} d \Psi_{0}$, since the pullback commutes with the exterior derivative. This corresponds to the usual frozen-in condition of ideal motion in the sense that the magnetic flux between pairs of advected points is preserved

$\Psi_{0}(b)-\Psi_{0}(a)=\int_{\gamma} d \Psi_{0}=\int_{\varphi(\gamma)} d \Psi=\Psi(\varphi(b))-\Psi(\varphi(a))$.

This integral would correspond to the poloidal magnetic flux in toroidal confinement devices [37] or helical magnetic flux in the vicinity of rational surfaces [39].

It is not required that the initial flux-function $\Psi_{0}$ : $M \rightarrow \mathbb{R}$ be smooth. However, if $\Psi_{0} \in C^{\infty}(M)$, as in the HKT problem, the condition that the advected fluxfunction be smooth, $\Psi \in C^{\infty}(M)$, is necessary for the configuration to be accessible through ideal motion from $\Psi_{0}$. In particular, the level-sets of $\Psi$ are diffeomorphic to those of $\Psi_{0}$, so that a configuration with no pre-existing magnetic islands cannot spontaneously develop islands 
when advected by smooth plasma motion (which is actually true regardless of whether the ideal plasma is in equilibrium or not).

The Hamiltonian vector field $B$ related to the frozen-in flux-function $\Psi=\varphi_{*} \Psi_{0}$ via $i_{B} \omega=d \Psi$ is in general not the same as the pushforward of the Hamiltonian vector field $B_{0}$ related to $\Psi_{0}$ by $i_{B_{0}} \omega=d \Psi_{0}$. It is only when the diffeomorphism $\varphi$ is volume-preserving, and incidentally a symplectomorphism, $\varphi^{*} \omega=\omega$, that the Poissonbracket is preserved $\varphi^{*}\{F, G\}=\left\{\varphi^{*} F, \varphi^{*} G\right\}$ (see Result B.2) and the magnetic fields $B$ and $B_{0}$ are $\varphi$-related (see Appendix A), namely $B=X_{\Psi}=X_{\varphi_{*} \Psi_{0}}=\varphi_{*} X_{\Psi_{0}}=$ $\varphi_{*} B_{0}$. This property means that the field-lines are tied to the fluid displacement. Volume-preserving diffeomorphisms/symplectomorphisms thus represent smooth incompressible ideal motion in this context.

The set of volume-preserving diffeomorphisms, denoted $S \operatorname{Diff}(M)$, has the structure of an infinite dimensional Lie-Fréchet group. Arnold famously exploited this construct to interpret the Euler equations as geodesic equations [24]. Ideal MHD has similar interpretations on semi-direct products [43-46].

We restrict our attention to the identity component $S \operatorname{Diff}^{0}(M)$, ruling out parity transformations for instance. The diffeomorphism $\varphi \in \operatorname{SDiff}^{0}(M)$, seen as a point on a manifold, is connected to the identity by a smooth path, namely a family of diffeomorphisms $\varphi_{t} \in S \operatorname{Diff}^{0}(M)$ for $t \in[0,1]$ with $\varphi_{0}=i d$ and $\varphi_{1}=\varphi$. The family $\varphi_{t}$ generates, in turn, a smooth family of fluxfunctions via the frozen-in condition (6)

$$
\Psi_{t}:=\Psi_{0} \circ \varphi_{t}^{-1}=\varphi_{t *} \Psi_{0} .
$$

The variation of $\varphi_{t}$ with respect to the parameter $t$ define a smooth family of vector fields on $M$ via

$$
X_{t}:=\partial_{t} \varphi_{t} \circ \varphi_{t}^{-1}: M \rightarrow T M
$$

called the Eulerian velocity field. The picture is that the trajectory of a fluid element, initially at $p_{0} \in M$, is $p(t)=\varphi_{t}\left(p_{0}\right)$, and its velocity is equal to the vector $d p / d t=\partial_{t} \varphi_{t}\left(p_{0}\right)=X_{t}(p(t))$. In local coordinates $p(t)=$ $(x(t), y(t))$, this corresponds to the following system of ordinary differential equations

$$
\dot{x}(t)=X^{x}(x(t), y(t), t), \quad \dot{y}(t)=X^{y}(x(t), y(t), t) .
$$

with $x(0)=x_{0}$ and $y(0)=y_{0}$ as initial conditions. It is worth mentioning that, for every initial point, existence and uniqueness of solutions over finite time is guaranteed by the Picard-Lindelöf theorem. This shows that the diffeomorphism $\varphi_{t}$ can be constructed as long as the vector field $X_{t}$ is smooth (Lipschitz continuous would suffice).

Because each diffeomorphism $\varphi_{t}$ is volume-preserving, $\varphi_{t}^{*} \omega=\omega$, the one-forms $i_{X_{t}} \omega$ are closed on $M$, implying that every vector field $X_{t}$ is divergence-free. Indeed, $0=\partial_{t} \omega=\partial_{t} \varphi_{t}^{*} \omega=\varphi_{t}^{*} £_{X_{t}} \omega$. Thus, $£_{X_{t}} \omega=$ $d i_{X_{t}} \omega=\left(\operatorname{div} X_{t}\right) \omega=0, \forall t$. The Hodge decomposition theorem [47] states that closed one-forms are uniquely representable as $i_{X_{t}} \omega=d S_{t}+K_{t} d y$, where $S_{t}(x, y)$ are smooth single-valued potential functions, and $K_{t}$ are coefficients (constant on $M$ ). In local coordinates, the Eulerian velocity field is expressed as

$$
X_{t}=\left(\partial_{y} S_{t}+K_{t}\right) \partial_{x}-\left(\partial_{x} S_{t}\right) \partial_{y}
$$

which is automatically divergence-free. This corresponds to $\boldsymbol{X}_{t}=\nabla \times\left[\left(S_{t}+K_{t} y\right) \nabla z\right]$ in the 3D picture. In local coordinates, the system of ODEs becomes

$$
\begin{aligned}
& \dot{x}(t)=\partial_{y} S(x(t), y(t), t)+K(t) \\
& \dot{y}(t)=-\partial_{x} S(x(t), y(t), t) .
\end{aligned}
$$

In this two-dimensional slab, divergence-free Eulerian vector fields fortunately coincide with Hamiltonian vector fields via $i_{X_{t}} \omega=d H_{t}$, where $H_{t}=S_{t}+K_{t} y$ is the family of generating functions. Here, $y$ is a locally-defined function such that $e^{i y}=w$ for $w \in S^{1}$. The choice of $y$ does not affect the Eulerian field nor the Poisson-bracket because different choices differ by a constant.

By differentiating the frozen-in condition (7) and using Result C.1, the flux-function is shown to satisfy the advection equation

$$
\partial_{t} \Psi_{t}=-X_{t} \Psi_{t}=\left\{S_{t}+K_{t} y, \Psi_{t}\right\} .
$$

When the Hamiltonian functions $H_{t}$ are given, this is a linear ordinary differential equation with variable coefficients, for which direct methods of solutions are known.

Unless $\varphi_{t}$ is a family of global isometries (rigid translations and rotations), the Laplacian $\Delta \Psi_{t} \neq \varphi_{t *} \Delta \Psi_{0}$, is not an advected quantity. This implies that the forcebalance condition $\left\{\Delta \Psi_{t}, \Psi_{t}\right\}=0$ will be respected only for very special cases of volume-preserving diffeomorphism $\varphi_{t}$. This statement is illustrated in the next section by considering one-parameter subgroups of diffeomorphisms generated by $t$-independent potential functions.

\section{A. Flow-maps and advection through $t$-independent potential functions}

In the case where the potential functions $S_{t}=S$ and coefficients $K_{t}=K$ are independent of $t$, the family of diffeomorphisms $\varphi_{t}$ coincides with the flow-map of the fixed vector field $X$ on $M$. Such families form one-parameter subgroups of $S \operatorname{Diff}^{0}(M)$ in the sense that $\varphi_{t} \circ \varphi_{s}=\varphi_{t+s}$. In this case, the solution to the advection equation (11) can be formally computed as

$$
\begin{aligned}
\Psi_{t} & =\exp (t\{H, .\}) \Psi_{0} \\
& =\Psi_{0}+t\left\{H, \Psi_{0}\right\}+\frac{t^{2}}{2}\left\{H,\left\{H, \Psi_{0}\right\}\right\}+\ldots
\end{aligned}
$$

where $H=S+K y$. The potential function $H$ generates a near-identity (canonical) transformation. Evaluated at any point $p=(x, y)$, such expansion is analytic in $t$ when it converges. 


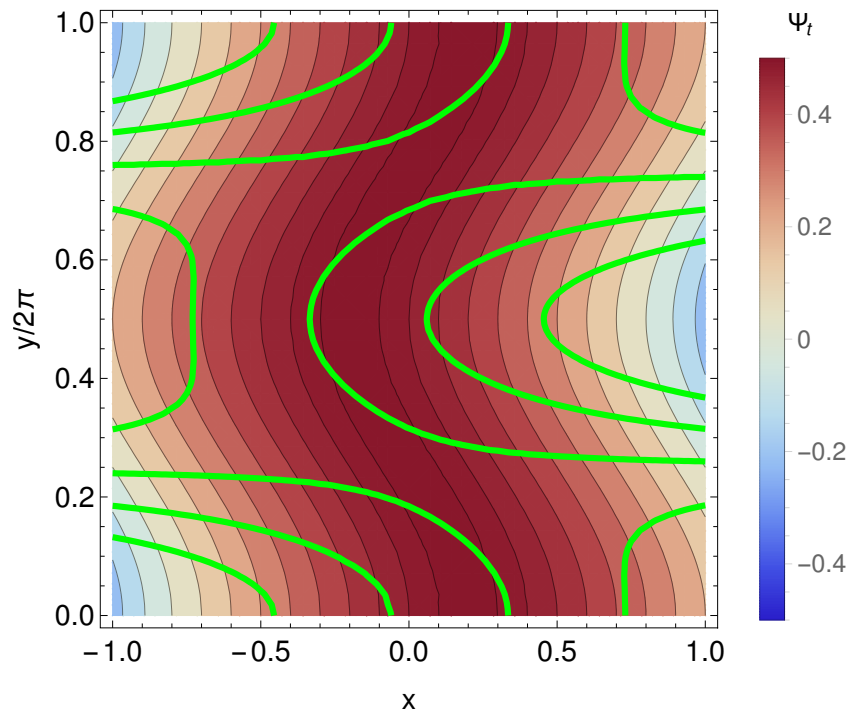

Figure 2. Advected flux $\Psi_{t}(p)=\Psi_{0}\left[\varphi_{t}^{-1}(p)\right]$ generated by the $t$-independent potential function $S(x, y)=\sin y$ for $t=0.2$ from initial HKT configuration $\Psi_{0}(x, y)=\frac{1}{2}\left(1-x^{2}\right)$. The colour contours represent the level sets of the flux-function and the thick green curves are level sets of the Laplacian $\Delta \Psi_{t}$. The fact that the two do not overlap indicates lack of force balance.

For example, consider the initial HKT configuration $\Psi_{0}(x, y)=\frac{1}{2}\left(1-x^{2}\right)$. We highlight the smooth ideal motion generated by the $t$-independent potential function $S(x, y)=\sin y$ and $K=0$. The corresponding system of ODEs for the coordinates has solution

$$
\mid \begin{array}{l|l}
\dot{x}=\cos y & \quad \\
\dot{y}=0
\end{array} \quad \begin{aligned}
& x(t)=x_{0}+t \cos y_{0} \\
& y(t)=y_{0}
\end{aligned}
$$

The advected flux-function is thus

$$
\begin{aligned}
\Psi_{t}(x, y) & =\Psi_{0}(x-t \cos y, y)=\frac{1}{2}\left[1-(x-t \cos y)^{2}\right] \\
& =\Psi_{0}(p)+t x \cos y-\frac{1}{2} t^{2} \cos ^{2} y
\end{aligned}
$$

which matches the expansion in equation (12) with $\left\{\sin y, \Psi_{0}\right\}=-\frac{1}{2}\left\{\sin y, x^{2}\right\}=x \cos y,\{\sin y, x \cos y\}=$ $-\cos ^{2} y$ and $\left\{\sin y, \cos ^{2} y\right\}=0$. The level sets of the advected flux-function are shown on Figure 2 for $t=0.2$. The flux-levels remain unbroken for all values $t$ by virtue of the frozen-in condition. This configuration is not in force balance since these level sets of the flux-function do not align with that of its Laplacian,

$$
\Delta \Psi_{t}=1+t x \cos y-t^{2} \cos (2 y)
$$

depicted by the green thick lines on Figure 2. The obstruction occurs at linear order in $t$, with the left-hand side of equation (5) being

$$
\left\{\Delta \Psi_{t}, \Psi_{t}\right\}=-t x^{2} \sin y+O\left(t^{2}\right)
$$

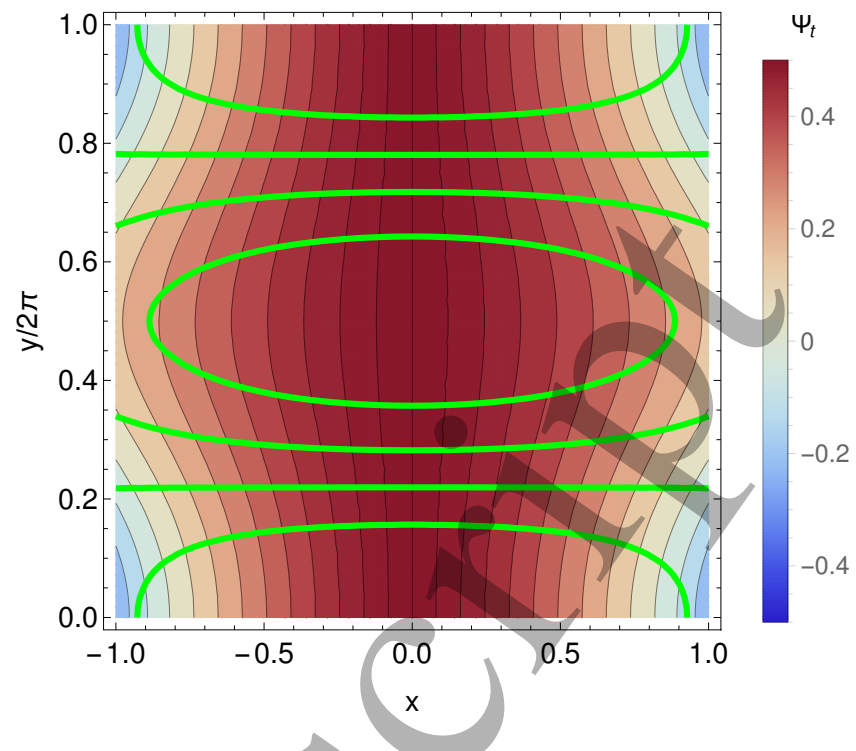

Figure 3. Same caption as Figure 2 but for generating potential function $S(x, y)=-x \sin y$.

A more intricate example is the flow-map generated by the potential function $S(x, y)=-x \sin y$ and $K=0$. The reader may verify that the coordinate transformation satisfies

$\mid \begin{aligned} & \dot{x}=-x \cos y \\ & \dot{y}=\sin y\end{aligned} \Longleftrightarrow \quad \begin{aligned} & x(t)=x_{0}\left(\cosh t-\sinh t \cos y_{0}\right) \\ & y(t)=2 \tan ^{-1}\left[e^{t} \tan \left(\frac{y_{0}}{2}\right)\right]\end{aligned}$

Rather neatly, the inverse map is obtained by negating the $t$-parameter such that the advected flux becomes

$$
\Psi_{t}(x, y)=\frac{1}{2}\left[1-x^{2}(\cosh t+\sinh t \cos y)^{2}\right] .
$$

It can be verified that the Taylor series in $t$ around $t=0$ of this flux-function matches the result from the expansion in equation (12). The level sets are shown on Figure 3 for $t=0.2$. The flux-levels remain unbroken for all values $t$ by the advection equation. This particular choice of potential function $S(x, y)$ preserves the mirror symmetry of the flux-function $\Psi_{t}(-x, y)=\Psi_{t}(x, y)$. This configuration is however not in force balance since the level sets of the flux-function do not align with that of its Laplacian, depicted by the green thick lines on Figure 3. The obstruction occurs again at linear order in $t$ with the left-hand side of equation (5) being equal to

$$
\left\{\Delta \Psi_{t}, \Psi_{t}\right\}=-t x\left(2-x^{2}\right) \sin y+O\left(t^{2}\right) .
$$

These examples suggest that the MHD equilibrium problem in a slab be viewed as the process of tuning the potential functions $S_{t}(x, y)$ until the level sets of the generated flux-functions and their Laplacian overlap for all $t$. In many respects, this is the point of view adopted by the Variational Moments Equilibrium Code (VMEC) [6], where three-dimensional magnetic configurations are obtained by minimising $\boldsymbol{J} \times \boldsymbol{B}-\nabla p$ through a steepestdescent method on the Fourier coefficients parametrising the (pre-existing) nested flux-surfaces. 


\section{SERIES SOLUTION}

The following strategy is proposed to construct the isotopy $\varphi_{t}$ : we expand $H_{t}=H_{0}+t H_{1}+\frac{1}{2} t^{2} H_{2}+\ldots$ in a Taylor series at $t=0$ and solve for the functions $H_{j}=$ $\left.\partial_{t}^{(j)} H_{t}\right|_{t=0}$ order-by-order via the force-balance condition $\left\{\Delta \Psi_{t}, \Psi_{t}\right\}=0$, where $\Psi_{t}=\Psi_{0}+t \Psi_{1}+\frac{1}{2} t^{2} \Psi_{2}+\ldots$ is the solution to the advection equation $\partial_{t} \Psi_{t}=\left\{H_{t}, \Psi_{t}\right\}$. Collecting terms, this yields a hierarchy of linear elliptic boundary-value problems

1. $\left\{\Delta \Psi_{1}, \Psi_{0}\right\}+\left\{\Delta \Psi_{0}, \Psi_{1}\right\}=0, \Psi_{1}=\left\{H_{0}, \Psi_{0}\right\}$.

2. $\left\{\Delta \Psi_{2}, \Psi_{0}\right\}+2\left\{\Delta \Psi_{1}, \Psi_{1}\right\}+\left\{\Delta \Psi_{0}, \Psi_{2}\right\}=0$, $\Psi_{2}=\left\{H_{1}, \Psi_{0}\right\}+\left\{H_{0}, \Psi_{1}\right\}$.

$$
\text { i. } \begin{aligned}
\sum_{j=0}^{i}\left(\begin{array}{c}
i \\
j
\end{array}\right)\left\{\Delta \Psi_{i-j}, \Psi_{j}\right\}=0, \\
\quad \Psi_{i}=\sum_{j=0}^{i-1}\left(\begin{array}{c}
i-1 \\
j
\end{array}\right)\left\{H_{i-1-j}, \Psi_{j}\right\} .
\end{aligned}
$$

At the $(i+1)^{t h}$ step, the force-balance equation is first used to determine $\Psi_{i+1}$, then $H_{i}$ is recovered by inverting its Poisson-bracket with $\Psi_{0}$. Formally, the $(i+1)^{t h}$ step consists of solving the following in-homogeneous linear system

$$
\begin{aligned}
\left(\operatorname{ad}_{\Psi_{0}} \Delta-\operatorname{ad}_{\Delta \Psi_{0}}\right) \Psi_{i+1} & =-\sum_{j=1}^{i}\left(\begin{array}{c}
i+1 \\
j
\end{array}\right)\left\{\Delta \Psi_{i+1-j}, \Psi_{j}\right\} \\
\operatorname{ad}_{\Psi_{0}} H_{i} & =\Psi_{i+1}-\sum_{j=1}^{i}\left(\begin{array}{l}
i \\
j
\end{array}\right)\left\{H_{i-j}, \Psi_{j}\right\}
\end{aligned}
$$

where the right-hand sides are known from previous steps, and $\operatorname{ad}_{\Psi_{0}}=B_{0}: C^{\infty}(M) \rightarrow C^{\infty}(M), F$ $\operatorname{ad}_{\Psi_{0}} F:=\left\{F, \Psi_{0}\right\}=B_{0} F$ is seen as a linear operator on functions. The system above is in a fairly suitable form for numerical implementations (spectral or finite-element methods).

Assuming that the problem can be set up on an appropriate vector space of functions, solvability conditions would be dictated by the Fredholm alternative; we expect solutions to exist if and only if the right-hand side of equations (13-14) does not lie within the Kernel of the linear operators on the left-hand side.

Specifically, among other requirements, the right-hand side of equation (14) must vanish on the set of critical points of $\Psi_{0}, \mathcal{C}_{0}=\left\{p \in M \mid d \Psi_{0 p}=0\right\}$, because the lefthand side is identically zero, $\left.\operatorname{ad}_{\Psi_{0}} F\right|_{\mathcal{C}_{0}} \equiv 0, \forall F$. Consequently, the value of $\Psi_{i+1}$ is determined on $\mathcal{C}_{0}$ by terms from the previous steps, independently of boundary conditions. In effect, this over-determines the boundaryvalue problem of equation (13), which bears solutions only if the boundary deformations do not interfere with the advection of the critical points of the initial condition $\Psi_{0}$. As seen in the next sections, this is the main obstruction to smoothly deforming equilibria through incompressible ideal MHD motion.

\section{A. Deforming vacuum fields}

We illustrate the procedure by applying it to the case where $\Psi_{0}$ has no critical points. To make the discussion as simple as possible, let us consider the one-dimensional flux-function $\Psi_{0}(x, y)=-x$ with straight level sets. The initial configuration

$$
d \Psi_{0}=-d x, \quad \star d \Psi_{0}=-d y, \quad B_{0}=\partial_{y}, \quad \Delta \Psi_{0}=0,
$$

satisfies the force balance condition. It is in fact a vacuum field (no current).

In this case, $\operatorname{ad}_{\Delta \Psi_{0}} \equiv 0$ is the zero-map and $\operatorname{ad}_{\Psi_{0}}=\partial_{y}$. Writing equations (13-14) for $i=0$, we thus have

$$
\partial_{y} \Delta \Psi_{1}=0, \quad \partial_{y} H_{0}=\Psi_{1},
$$

where $\Psi_{1}=\left.\partial_{t} \Psi_{t}\right|_{t=0}$ is interpreted as the initial rate of change of $\Psi_{t}$ along the path $\varphi_{t}$. Force-balance requires this quantity to be of the form

$$
\Psi_{1}=h(x, y)+f(x)
$$

where, by standard separation of variables,

$$
\begin{gathered}
h(x, y)=\sum_{n=1}^{\infty}\left[A_{n} \cosh (n x) \cos (n y)+B_{n} \cosh (n x) \sin (n y)\right. \\
\left.+C_{n} \sinh (n x) \cos (n y)+D_{n} \sinh (n x) \sin (n y)\right]
\end{gathered}
$$

is a harmonic function on $M$ with $A_{n}, B_{n}, C_{n}$ and $D_{n}$ determined by boundary conditions.

The potential function $H_{0}=S_{0}+K_{0} y$ then satisfies

$$
\partial_{y} S_{0}=h+f-K_{0} .
$$

Since $\oint \partial_{y} S_{0} d y=0$ and $\oint h d y=0$, the function $f$ is deduced, after integrating equation (18) over y, to be constant $f(x)=K_{0}$. The initial rate of change is thus a purely harmonic function $\Psi_{1}=h+K_{0}$ and the potential function has the form

$$
S_{0}(x, y)=k(x, y)+g(x)
$$

where

$$
\begin{gathered}
k(x, y)=\sum_{n=1}^{\infty} \frac{1}{n}\left[A_{n} \cosh (n x) \sin (n y)-B_{n} \cosh (n x) \cos (n y)\right. \\
\left.+C_{n} \sinh (n x) \sin (n y)-D_{n} \sinh (n x) \cos (n y)\right]
\end{gathered}
$$

is a harmonic function such that $\partial_{y} k=h$. We note that the $g(x)$ term does not contribute to displacing flux-levels at $t=0$, since $B_{0} g=\partial_{y} g=0$. The potential function above leads to the following Eulerian velocity field

$$
X_{0}=\left(h+K_{0}\right) \partial_{x}-\left(\partial_{x} k+g^{\prime}\right) \partial_{y}
$$


We note that the perturbed flux-function $\Psi_{t}=\Psi_{0}+$ $t \Psi_{1}+O\left(t^{2}\right)$ still describes a vacuum field at first-order.

At the next order, equations (13-14) with $i=1$ become

$$
\begin{aligned}
\partial_{y} \Delta \Psi_{2} & =-2\left\{\Delta \Psi_{1}, \Psi_{1}\right\}=0, \\
\partial_{y} H_{1} & =\Psi_{2}-\left\{H_{0}, \Psi_{1}\right\}=\Psi_{2}-X_{0} h
\end{aligned}
$$

The solution of the first equation is again in the form $\Psi_{2}=h_{2}(x, y)+f_{2}(x)$ where $h_{2}$ is a harmonic function as in equation (17) with different coefficients. The potential function $H_{1}=S_{1}+K_{1} y$ then satisfies

$\partial_{y} S_{1}=h_{2}+f_{2}-K_{1}-\partial_{x} h^{2}+\partial_{y}\left(h \partial_{x} k\right)-K_{0} \partial_{x} h+g^{\prime} \partial_{y} h$

where $\partial_{y} k=h$ was used to write $\left(\partial_{x} k\right)\left(\partial_{y} h\right)=$ $\partial_{y}\left(h \partial_{x} k\right)-h \partial_{x} h$. Averaging over $y$, the function $f_{2}$ is deduced to be

$$
f_{2}(x)=K_{1}+\frac{1}{2 \pi} \partial_{x} \oint h^{2} d y
$$

The explicit expression of $S_{1}$ is omitted. We note that the flux-function may develop current at second-order, since $\Delta \Psi_{t}=\frac{1}{2} t^{2} \Delta \Psi_{2}+O\left(t^{3}\right)=\frac{t^{2}}{4 \pi} \partial_{x}^{3} \oint h^{2} d y+O\left(t^{3}\right)$.

It is seen that, aside from becoming increasingly tedious, there is no major obstruction to pursuing the calculation at higher orders.

\section{B. Hahm-Kulsrud-Taylor (HKT) problem}

Let us now consider the initial flux-function $\Psi_{0}(x, y)=$ $\frac{1}{2}\left(1-x^{2}\right)$, also with straight flux-levels. The configuration

$$
d \Psi_{0}=-x d x, \quad \star d \Psi_{0}=-x d y, \quad B_{0}=x \partial_{y}, \quad \Delta \Psi_{0}=1,
$$

satisfies the force balance condition and represents an initial MHD equilibrium in the vicinity of a resonant surface. This configuration features a line of degenerate critical points, $\mathcal{C}_{0}=\{(x, y) \mid x=0\}$, restricted to which $\operatorname{ad}_{\Psi_{0}} \mid \mathcal{C}_{0} \equiv 0$. Consequently, the left-hand side of equation (14) evaluated on $\mathcal{C}_{0}$ vanishes. The value of $\Psi_{i+1} \mid \mathcal{C}_{0}=$ $\Psi_{i+1}(0, y)$ must match that of $\sum_{j=1}^{i}\left(\begin{array}{l}i \\ j\end{array}\right)\left\{H_{i-j}, \Psi_{j}\right\} \mid \mathcal{C}_{0}$ from previous steps. This solvability condition acts as an additional boundary condition in equation (13).

For $i=0$, equations (13-14) dictate the initial rate of change $\Psi_{1}=\left.\partial_{t} \Psi_{t}\right|_{t=0}$ along $\varphi_{t}$ together with the generating potential function $H_{0}=S_{0}+K_{0} y$. We have

$$
\partial_{y} \Delta \Psi_{1}=0, \wedge \quad x \partial_{y} H_{0}=\Psi_{1} .
$$

The first equation is solved by

$$
\Psi_{1}=h(x, y)+f(x)
$$

where $h$ is a harmonic function as in equation (17). The potential function is then found as

$$
\partial_{y} S_{0}=\frac{h}{x}+\frac{f}{x}-K_{0}
$$

Since $\oint \partial_{y} S_{0} d y=0$ and $\oint h d y=0$, the function $f$ is seen, after integrating equation (28) over $y$, to be of the form $f(x)=K_{0} x$. The initial rate of change is thus a purely harmonic function $\Psi_{1}=h+K_{0} x$ and the potential function has the form

$$
S_{0}(x, y)=\frac{k(x, y)}{x}+g(x)
$$

where $k$ is a harmonic function in the same form as equation (20). The potential function above leads to the following Eulerian velocity field

$$
X_{0}=\left(\frac{h}{x}+K_{0}\right) \partial_{x}+\left(\frac{k-x \partial_{x} k}{x^{2}}-g^{\prime}\right) \partial_{y} .
$$

Solvability of the second equation of (26) requires the initial rate of change to vanishes on the neutral line $\mathcal{C}_{0}$, namely $\Psi_{1}(0, y)=h(0, y)=0$. This condition implies $A_{n}=0$ and $B_{n}=0$, which is necessary for smooth incompressible ideal motion. If $A_{n}$ and $B_{n}$ were non-zero, the potential function and the Eulerian velocity field would become singular and it would be impossible to construct the family of diffeomorphisms $\varphi_{t}$ through the ODE system (10). The boundary conditions on $\Psi_{t}$ must thus be compatible with the fact that the initial rate of change $\Psi_{1}$ can only be made an odd function of $x$.

In fact, we prove by strong induction that it is impossible to deform the plasma such that the mirror symmetry of the flux-function is preserved, $\Psi_{t}(-x, y)=\Psi_{t}(x, y)$, at all orders in $t$. Deformations that preserve the evenparity of the flux-function (mirror symmetry) would be referred to as tearing-like response in the context of resistive MHD stability [48, 49], whereas odd-parity displacements would be called kink-like response [50].

The base case consists of the fact that if mirror symmetry were to be preserved, $C_{n}=0, D_{n}=0$ and $K_{0}=0$, namely the initial rate of change vanishes entirely, $\Psi_{1}=\left.\partial_{t} \Psi_{t}\right|_{t=0}=0$. Let us assume that mirror symmetry of the flux-function is respected up to its $i^{\text {th }}$ derivative in $t$ at $t=0$, i.e. $\Psi_{j}=\left.\partial_{t}^{(j)} \Psi_{t}\right|_{t=0}=0$ for $j=1, \ldots i$. Equation (13) then yields

$$
\partial_{y} \Delta \Psi_{i+1}=-\sum_{j}^{i}\left(\begin{array}{c}
i+1 \\
j
\end{array}\right)\left\{\Delta \Psi_{i+1-j}, \Psi_{j}\right\}=0
$$

i.e. $\Psi_{i+1}=h_{i+1}(x, y)+f_{i+1}(x)$, similarly to equation (27). Equation (14) reduces to

$$
x\left(\partial_{y} S_{i}+K_{i}\right)=\Psi_{i+1}
$$

Integrating over $y$, the function $f_{i+1}$ must be of the form $f_{i+1}(x)=K_{i} x$, the only even-parity preserving choice however being $K_{i}=0$. As before, the solvability of equation (32) imposes the boundary condition $\Psi_{i+1}(0, y)=0$, thereby eliminating all even-parity terms from the harmonic function $h_{i+1}$. We thus conclude that $\Psi_{i+1}=0$. By induction, this result extends to all derivatives of the flux-function with respect to $t$ at $t=0$. 
If there exists a smooth family of diffeormophisms such that the advected flux-function remains even in $x$ and in force balance for all values of the time-like parameter $t$, then it is non-analytic in $t$ at $t=0$. Conversely, a path of diffeormorphisms $\varphi_{t}$, analytic in $t$ at $t=0$ and such that $\Psi_{t}$ is in force balance, does not preserve the even-parity of the flux-function. In particular, mirrorsymmetric boundary conditions such as

$$
\Psi_{t}( \pm a, y)=\Psi_{0}(a)\left[1+t^{i} \cos (n y+\alpha)\right]
$$

are unsupported for all $t$ and all integer power $i \geq 1$ $(\forall n \in \mathbb{N}, \forall \alpha \in[0,2 \pi])$. Consequently, the time-like variable $t$ cannot serve as an expansion parameter to control the application of mirror-symmetric boundary perturbations. In other words, the HKT initial equilibrium is rigid to smooth ideal deformations that preserve the even property of the flux-function with respect to the neutral line.

The above result does not rule out the possibility of connecting the initial HKT configuration to a paritypreserving state with curved level sets via a path of diffeomorphisms that is non-analytic with respect to the control parameter $t$. Such ideal motion would be quite special from the point of view of a continuous deformation of the boundary. In fact, formulated as an over-determined boundary value problem $-\Delta \Psi=V^{\prime}(\Psi)$ with $d \Psi=0$ at $x=0$ and $\Psi=0$ on the edge of an mirror-symmetric domain in $x$, the existence (and regularity) of solutions with non-straight level sets is rather unlikely [51]. The details of this question will be addressed in future work.

\section{Flow-map of equilibrium-preserving generating potential function}

A typical example of a smooth generating potential function respecting force-balance at $t=0$ would be

$$
S_{0}(x, y)=\frac{\sinh x}{x} \sin y .
$$

Using this potential function in equation (12) to compute the flow-map of a $t$-independent vector field, the advected flux-function is reconstructed as

$$
\begin{aligned}
\Psi_{t}=\Psi_{0} & +t \sinh x \cos y \\
& +t^{2} \frac{\sinh x\left(\sinh x \sin ^{2} y-x \cosh x\right)}{2 x^{2}}+O\left(t^{3}\right),
\end{aligned}
$$

whose level sets are shown in Figure 4. The green lines, depicting level sets of the Laplacian $\Delta \Psi_{t}$, still do not align with the flux-levels, however, the term spoiling force-balance is now of order $O\left(t^{2}\right)$, as can be checked by computing $\left\{\Delta \Psi_{t}, \Psi_{t}\right\}$. There is no obstruction to continuing the procedure at the next order to derive $S_{t}=S_{0}+t S_{1}$ such that $\left\{\Delta \Psi_{t}, \Psi_{t}\right\}=O\left(t^{3}\right)$ (omitted here for brevity).

The fact that force-balance could not be achieved through a $t$-independent vector field suggests that an

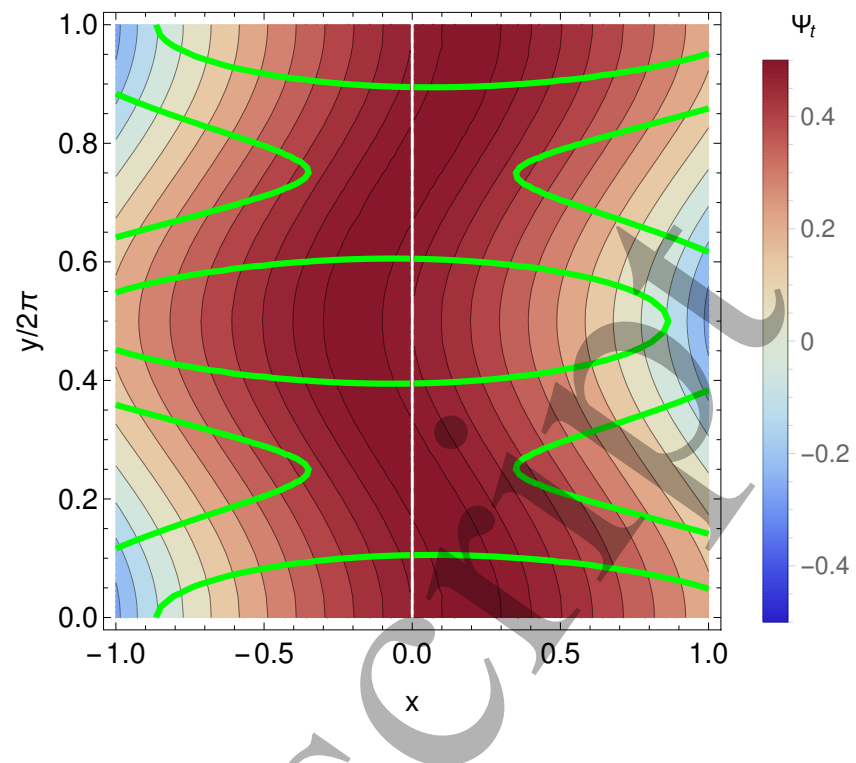

Figure 4. Advected flux $\Psi_{t}(p)$ generated by the $t$-independent potential function $S(x, y)=\sinh x \sin y / x$ for $t=0.2$ from initial HKT configuration $\Psi_{0}(x, y)=\frac{1}{2}\left(1-x^{2}\right)$, computed using equation (12) up to fifth order. The colour contours represent the level sets of the flux-function and the thick green curves are level sets of the Laplacian $\Delta \Psi_{t}$. The fact that the two do not overlap indicates lack of force balance. The obstruction is however quadratic in $t$, instead of linear as in figures 2 and 3.

equilibrium-preserving diffeomorphism has to be generated by time-dependent potential functions. Smooth ideal incompressible motion from an initial equilibrium to another accessible state is most likely not in any oneparameter group of diffeomorphisms. This is to be expected by the non-linearity of the algebraic force-balance condition in contrast to the advection equation.

\section{DISCUSSION}

In this paper, we addressed the problem of accessing MHD equilibrium states via smooth ideal motion and highlighted the main obstruction to finding a smooth family of force-balanced flux-functions in the simplest two-dimensional slab case known as the Hahm-KulsrudTaylor problem. The MHD force-balance condition was conveniently written as the Poisson-commutation of the flux-function $\Psi_{t}$ with its Laplacian $\Delta \Psi_{t}$. This form was achieved in the local limit of a flux tube or whenever a Grad-Shafranov equation holds (namely when a symmetry by isometry is present). The strategy was then to find a family of volume-preserving diffeomorphisms to advect an initial equilibrium configuration into another with deformed but topologically-equivalent flux-levels. The use of volume-preserving maps (symplectomorphisms) conveniently insured invariance of the Poisson-bracket and the freezing of magnetic field-lines in the fluid displacement. The problem was then to solve for the family of generat- 
ing potential functions $S_{t}$, which are in effect $t$-dependent Hamiltonian functions for the Eulerian velocity field $X_{t}$. Starting with the one-dimensional HKT equilibrium configuration, the initial rate of change $\Phi=\left.\partial_{t} \Psi_{t}\right|_{t=0}$ of the flux-function was found to be a harmonic function. However, through its relation to the generating function, the initial rate of change had to vanish on the line of critical points of $\Psi_{0}=\frac{1}{2}\left(1-x^{2}\right)$ at $x=0$. This condition excluded the application of boundary conditions that retain the mirror symmetry of the flux-function. It was proved by strong induction on the Taylor expansion in $t$ that those boundary conditions had to be excluded at all orders.

This result showed that, within the class of smooth solutions and via ideal motion (perfect advection of flux), some MHD equilibria do not deform when subject to certain boundary perturbations. This rigidity implies from a computational and numerical point of view that at least one of the constraints on the motion must be relaxed in order to generate solutions, e.g. allowing for discontinuities, including the effect of finite resistivity (dissipate flux accumulation), enabling regions of force imbalance, etc. Deciding which workaround is more suitable is left for debate.

Relaxing the hypothesis of smooth ideal motion and allowing for discontinuities in the magnetic field circumvents the issue. Several methods of solution to the HKT problem [2, 31-36] allow the flux-function to become discontinuous at $x=0$, thereby introducing a current sheet proportional to the jump in $\left.\partial_{x} \Psi\right|_{x=0}$. In effect, the do- main is partitioned into two independent regions of forcebalance, where the neutral line acts on each side as a source or sink of flux. This is no longer the same as requiring flux advection by a global diffeomorphism, unless the discontinuity in the flux-function pre-exists in the initial configuration. Another example is the approach adopted by the SPEC [7] where the flux-function is continuous but not differentiable on a finite set of fluxlevels. The study of smooth deformation of force-free states is an interesting on-going research topic, in particular the question of avoidance of islands and stochastic fields through suitable boundary deformations.

It is well-understood that there always is a finite amount of resistivity in a physical system, that allows for profile smoothing and release of flux in infinitesimal regions around resonant surfaces (neutral line in the flux-function). This is reminiscent of d'Alembert's paradox in fluid dynamics, where the drag force vanishes in the limit of an inviscid potential flow, contradicting the phenomenological observation of substantial drag at high Reynolds numbers.

Without resistivity, the commonly accepted solution to the HKT problem is a boundary layer treatment originally proposed by Rosenbluth et al. [1] for the non-linear saturation of the internal kink instability. In a small region around the neutral line, a discontinuous solution is obtained with approximate force balance, which is then asymptotically matched with the ideal solution on the outside. This method is, in light of this paper, not to be mistaken with a strictly ideal treatment that mandates smoothness.
[1] M. N. Rosenbluth, R. Y. Dagazian, and P. H. Rutherford, The Physics of Fluids 16, 1894 (1973).

[2] A. H. Boozer and N. Pomphrey, Physics of Plasmas 17, 110707 (2010).

[3] R. B. White, Physics of Plasmas 20, 022105 (2013).

[4] J. Loizu and P. Helander, Physics of Plasmas 24, 040701 (2017).

[5] Y. Zhou, Y.-M. Huang, A. H. Reiman, H. Qin, and A. Bhattacharjee, Physics of Plasmas 26, 022103 (2019).

[6] S. P. Hirshman and J. C. Whitson, Physics of Fluids 26, 3553 (1983).

[7] S. R. Hudson, R. L. Dewar, G. Dennis, M. J. Hole, M. McGann, G. von Nessi, and S. Lazerson, Physics of Plasmas 19, 112502 (2012).

[8] Y. Zhou, H. Qin, J. W. Burby, and A. Bhattacharjee, Physics of Plasmas 21, 102109 (2014).

[9] K. Harafuji, T. Hayashi, and T. Sato, Journal of Computational Physics 81, 169 (1989).

[10] Y. Suzuki, N. Nakajima, K. Watanabe, Y. Nakamura, and T. Hayashi, Nuclear Fusion 46, L19 (2006).

[11] J. A. Krommes and A. H. Reiman, Physics of Plasmas (1994-present) 16, 072308 (2009).

[12] S. P. Hirshman, R. Sanchez, and C. R. Cook, Physics of Plasmas (1994-present) 18, 062504 (2011).

[13] C. Bressan, M. Kraus, P. J. Morrison, and O. Maj, Jour- nal of Physics: Conference Series 1125, 012002 (2018).

[14] M. Landreman and W. Sengupta, Journal of Plasma Physics 84, 905840616 (2018).

[15] M. Landreman, W. Sengupta, and G. G. Plunk, Journal of Plasma Physics 85, 905850103 (2019).

[16] D. A. Spong, S. P. Hirshman, J. C. Whitson, D. B. Batchelor, B. A. Carreras, V. E. Lynch, and J. A. Rome, Physics of Plasmas 5, 1752 (1998).

[17] A. Reiman, G. Fu, S. Hirshman, L. Ku, D. Monticello, H. Mynick, M. Redi, D. Spong, M. Zarnstorff, B. Blackwell, A. Boozer, A. Brooks, W. A. Cooper, M. Drevlak, R. Goldston, J. Harris, M. Isaev, C. Kessel, Z. Lin, J. F. Lyon, P. Merkel, M. Mikhailov, W. Miner, G. Neilson, M. Okamoto, N. Pomphrey, W. Reiersen, R. Sanchez, J. Schmidt, A. Subbotin, P. Valanju, K. Y. Watanabe, R. White, N. Nakajima, and C. Nührenberg, Plasma Physics and Controlled Fusion 41, B273 (1999).

[18] P. Helander, Reports on Progress in Physics 77, 087001 (2014).

[19] P. J. Morrison and J. M. Greene, Phys. Rev. Lett. 45, 790 (1980).

[20] D. D. Holm, J. E. Marsden, T. Ratiu, and A. Weinstein, Physics Reports 123, 1 (1985).

[21] J. R. Cary and R. G. Littlejohn, Annals of Physics 151, 1 (1983). 
[22] V. D. Shafranov, Journal of Nuclear Energy. Part C, Plasma Physics, Accelerators, Thermonuclear Research 5, 251 (1963).

[23] H. Grad, The Physics of Fluids 10, 137 (1967).

[24] V. I. Arnold, Annales de l'Institut Fourier 16, 319 (1966).

[25] M. Hénon, Comptes Rendus Acad. Sci. Paris A 262, 312 (1966).

[26] T. Dombre, U. Frisch, J. M. Greene, M. Hénon, A. Mehr, and A. M. Soward, Journal of Fluid Mechanics 167, 353-391 (1986).

[27] A. Lichtenberg and M. Lieberman, Regular and Chaotic Dynamics, Applied Mathematical Sciences (Springer New York, 2010).

[28] S. Abdullaev, Magnetic Stochasticity in Magnetically Confined Fusion Plasmas: Chaos of Field Lines and Charged Particle Dynamics, Springer Series on Atomic, Optical, and Plasma Physics (Springer, 2013).

[29] V. I. Arnold, Proceedings of the Summer School in Differential Equations, Erevan (1974).

[30] H. K. Moffatt, Journal of Fluid Mechanics 159, 359-378 (1985).

[31] T. S. Hahm and R. M. Kulsrud, The Physics of Fluids 28, 2412 (1985).

[32] E. G. Zweibel and H.-S. Li, Astrophysical Journal 312, 423 (1987).

[33] R. L. Dewar, A. Bhattacharjee, R. M. Kulsrud, and A. M. Wright, Physics of Plasmas 20, 082103 (2013).

[34] J. Loizu, S. Hudson, A. Bhattacharjee, and P. Helander, Physics of Plasmas 22, 022501 (2015).

[35] Y. Zhou, Y.-M. Huang, H. Qin, and A. Bhattacharjee, Phys. Rev. E 93, 023205 (2016).

[36] R. L. Dewar, S. R. Hudson, A. Bhattacharjee, and Z. Yoshida, Physics of Plasmas 24, 042507 (2017).

[37] W. D'Haeseleer, Flux coordinates and magnetic field structure: a guide to a fundamental tool of plasma structure, Springer series in computational physics (SpringerVerlag, 1991)

[38] A. H. Boozer, Rev. Mod. Phys. 76, 1071 (2005).

[39] L. E. Zakharov, Physics of Plasmas 15, 062507 (2008).

[40] H. R. Strauss, The Physics of Fluids 19, 134 (1976).

[41] M. Kraus, E. Tassi, and D. Grasso, Journal of Computational Physics 321, 435 (2016).

[42] J. B. Taylor, Phys. Rev. Lett. 33, 1139 (1974).

[43] J. E. Marsden, T. Ratiu, and A. Weinstein, Transactions of the American Mathematical Society 281, 147 (1984).

[44] D. D. Holm, J. E. Marsden, and T. S. Ratiu, Advances in Mathematics 137, 1 (1998).

[45] T. Ono, Physica D: Nonlinear Phenomena 81, 207 (1995).

[46] Y. Hattori, Journal of Physics A: Mathematical and General 27, L21 (1994).

[47] G. Schwarz, Hodge decomposition: A method for solving boundary value problems, Lecture notes in mathematics ; 1607. (Springer-Verlag, Berlin, 1995).

[48] H. P. Furth, J. Killeen, and M. N. Rosenbluth, The Physics of Fluids 6, 459 (1963).

[49] P. H. Rutherford, The Physics of Fluids 16, 1903 (1973).

[50] J.-k. Park, A. H. Boozer, J. E. Menard, A. M. Garofalo, M. J. Schaffer, R. J. Hawryluk, S. M. Kaye, S. P. Gerhardt, and S. A. Sabbagh, Physics of Plasmas 16, 056115 (2009).

[51] J. Serrin, Archive for Rational Mechanics and Analysis 43, 304 (1971).

[52] R. Abraham and J. E. Marsden, Foundations of mechan- ics, 2nd ed. (Addison-Wesley, 1978).

\section{Appendix A: Definitions and conventions from differential geometry}

The interior product (contraction) of a vector field $X \in \Gamma(T M)$ with a $k$-form $\alpha \in \Omega^{k}(M)$ is the unique $(k-1)$-form such that $\left[i_{X} \alpha\right]\left(Y_{1}, \ldots, Y_{k-1}\right)=$ $\alpha\left(X, Y_{1}, \ldots, Y_{k-1}\right) \in C^{\infty}(M), \forall Y_{1}, \ldots, Y_{k-1} \in \Gamma(T M)$.

The so-called musical isomorphism between vector fields and one-forms is established through the Riemannian metric (relation between co-variant and contravariant tensor fields). In particular, the flat of a vector field is the unique one-form such that $X^{\mathrm{b}}(Y)=\langle X, Y\rangle \in$ $C^{\infty}(M), \forall Y \in \Gamma(T M)$.

The Hodge star $\star$ operator is the isomorphism between $k$-forms and $n-k$ forms on $M$ with the property that for $\alpha, \beta \in \Omega^{k}(M), \alpha \wedge \star \beta=\langle\alpha, \beta\rangle \omega$, where $\omega \in \Omega^{n}(M)$ is the natural volume form associated to the Riemannian metric. The inner product on $k$-forms is extended from the Riemannian metric (Gram determinant). This convention implies $\star \omega=1$.

Given vector field $X \in \Gamma(T M)$, the Lie derivative of vector field $Y \in \Gamma(T M)$ is a vector field denoted by $£_{X} Y=[X, Y] \in \Gamma(T M)$ which acts on functions as $£_{X} Y f=X Y f-Y X f$. The Lie derivative of a $k$-form $\alpha \in \Omega^{k}(M)$ is defined accordingly. In practice though, one relies on Cartan's formula $£_{X} \alpha=i_{X} d \alpha+d i_{X} \alpha$. For functions (zero-forms), the Lie derivative along $X$ at point $p \in M$ reduces to the directional derivative $£_{X} f(p)=i_{X} d f(p)=X f(p)$.

As in [52, Definition 1.7.16], given a diffeomorphism $\varphi: M \rightarrow M$, the pushforward of vector field $X \in \Gamma(T M)$ is the vector field $\varphi_{*} X:=T \varphi \circ X \circ \varphi^{-1} \in \Gamma(T M)$ where $T \varphi: T M \rightarrow T M$ is the tangent map (Jacobian matrix in local coordinates). The pullback of a $k$-form $\alpha \in \Omega^{k}(M)$ is defined via $\left[\varphi^{*} \alpha\right]_{x}\left(v_{1}, \ldots, v_{k}\right)=$ $\alpha_{\varphi(x)}\left(T \varphi_{x}\left(v_{1}\right), \ldots, T \varphi_{x}\left(v_{k}\right)\right)$ where $v_{1}, \ldots, v_{k} \in T_{x} M$ are tangent vectors at $x \in M$. The important properties of the pullback is that it is compatible with the wedge product, $\varphi^{*}(\alpha \wedge \beta)=\varphi^{*} \alpha \wedge \varphi^{*} \beta$, and with the exterior derivative $\varphi^{*} d \alpha=d \varphi^{*} \alpha$.

The pullback of a vector field $X \in \Gamma(T M)$ refers to the pushforward-by-inverse, $\varphi^{*} X:=\varphi_{*}^{-1} X$. Accordingly, the "pushforward" of $k$-form $\alpha \in \Omega^{k}(M)$ is an alias for pullback-by-inverse, $\varphi_{*} \alpha:=\varphi^{-1^{*}} \alpha$.

\section{Appendix B: Poisson-brackets on symplectic manifolds}

A symplectic form $\omega \in \Omega^{2}(M)$ on $M$ is a 2 -form that is closed, $d \omega=0$, and non-degenerate, $i_{X} \omega=0 \Longleftrightarrow$ $X=0$. By the latter property, the exterior derivative of a smooth function $F: M \rightarrow \mathbb{R}$ uniquely determines a 
Hamiltonian vector field $X_{F} \in \Gamma(T M)$ via

$$
i_{X_{F}} \omega=d F
$$

Hamiltonian vector fields are generators of symplectomorphisms in the sense that the symplectic form is preserved under their flow. This property is reflected in

$$
£_{X_{F}} \omega=i_{X_{F}} d \omega+d i_{X_{F}} \omega=d^{2} F=0
$$

Since $X F=£_{X_{F}} F=i_{X_{F}} d F=i_{X_{F}} i_{X_{F}} \omega=0$, the function $F$ is preserved along the flow of its Hamiltonian vector field $X_{F}$. In other words, the vector field $X_{F}$ is tangential to the level sets of $F$.

Given a pair of smooth functions $F$ and $G$, the following anti-symmetric bilinear operation is defined

$$
\{F, G\}:=\omega\left(X_{F}, X_{G}\right)=-X_{F} G=X_{G} F .
$$

Result B.1. This product is a Poisson-bracket, namely it obeys Leibniz's rule

$$
\{F G, H\}=\{F, H\} G+F\{G, H\} .
$$

as well as the Jacobi identity

$$
\{F,\{G, H\}\}+\{G,\{H, F\}\}+\{H,\{F, G\}\}=0 .
$$

The Jacobi identity for the Poisson bracket then follows from the Jacobi identity satisfied by the Lie bracket of vector fields,

$$
X_{\{F,\{G, H\}\}+\mathrm{cycl}}=\left[X_{F},\left[X_{G}, X_{H}\right]\right]+\mathrm{cycl}=0 .
$$

Result B.2 (Preservation of the Poisson-bracket by symplectomorphism). Let $F, G \in C^{\infty}(M)$ and $X_{F}, X_{G} \in$ $\Gamma(T M)$ the corresponding Hamiltonian vector fields. Let $\varphi: M \rightarrow M$ be a symplectomorphism, $\varphi^{*} \omega=\omega$. Then, $X_{\varphi^{*} F}=\varphi^{*} X_{F}, X_{\varphi^{*} G}=\varphi^{*} X_{G}$ and $\varphi^{*}\{F, G\}=$ $\left\{\varphi^{*} F, \varphi^{*} G\right\}$.

Proof. Since the pullback commutes with the exterior derivative, $\varphi^{*} d F=d \varphi^{*} F$, it follows that

$$
\begin{aligned}
i_{\varphi^{*} X_{F}} \omega & =i_{\varphi * X_{F}} \varphi^{*} \omega=\varphi^{*}\left(i_{X_{F}} \omega\right) \\
& =\varphi^{*} d F=d \varphi^{*} F=i_{X_{\varphi^{*}}} \omega
\end{aligned}
$$

and likewise for $G$. Then,

$$
\begin{aligned}
\varphi^{*}\{F, G\} & =\varphi^{*}\left(\omega\left(X_{F}, X_{G}\right)\right)=\varphi^{*} \omega\left(\varphi^{*} X_{F}, \varphi^{*} X_{G}\right) \\
& =\omega\left(X_{\varphi^{*} F}, X_{\varphi^{*} G}\right)=\left\{\varphi^{*} F, \varphi^{*} G\right\} .
\end{aligned}
$$

\section{Appendix C: Advection equations}

In this section, we consider $\varphi_{t}: M \rightarrow M$ to be a smooth family of diffeomorphisms with corresponding Eulerian velocity field $X_{t}:=\partial_{t} \varphi_{t} \circ \varphi_{t}^{-1}$. We denote the curve $p(t)=\varphi_{t}\left(p_{0}\right) \in M$, with tangent velocity $\dot{p}(t)=X_{t}(p(t)) \in T M_{p(t)}$.

Result C.1. Let $\Psi_{t}:=\varphi_{t *} \Psi_{0} \in C^{\infty}(M)$ be a family of frozen-in functions. Then

$$
\partial_{t} \Psi_{t}=-X_{t} \Psi_{t}=-£_{X_{t}} \Psi_{t}
$$

Proof. The frozen-in condition evaluated along the curve reads $\Psi_{0}\left(p_{0}\right)=\Psi_{t}(p(t))$. Differentiating with respect to $t$, applying the Leibniz and chain rules, we have

$$
\begin{aligned}
0 & =\partial_{t} \Psi_{t}(p(t))+d \Psi_{t p(t)}\left(X_{t}(p(t))\right) \\
& =\partial_{t} \Psi_{t}(p(t))+\left[X_{t} \Psi_{t}\right](p(t)) .
\end{aligned}
$$

For every $t$, the point $p(t)=\varphi_{t}\left(p_{0}\right)$ can be made arbitrary by adjusting the initial condition (choose $p_{0}=\varphi_{t}^{-1} q$ ). Hence, $\forall t, \partial_{t} \Psi_{t}(q)=-\left[X_{t} \Psi_{t}\right](q), \forall q \in M$. 